\title{
OC27 - How to manage teenagers' emotions? Proposal of an algorithm for nursing intervention
}

\author{
Maria João Caeiro (Portugal)1,2; Paula Diogo (Portugal) ${ }^{1,3}$ \\ ${ }^{1}$ UI\&DE: Research Line 'Emotions in Health'; ${ }^{2}$ CHLC-Hospital D. Estefânia, Unidade \\ de Adolescentes; ${ }^{3}$ Escola Superior de Enfermagem de Lisboa, ESEL
}

Theme: Complex health care and chronic disease management.

Keywords: Emotional labour, hospitalization, paediatric nursing, teenagers.

The singular human experience of emotions is a relevant subject of nursing care. It determines not only the importance of emotions, but also the underlying knowledge required in nursing practice.

The algorithm aims at giving a directed answer according to clients' emotional responses, while mitigating or minimizing emotionally intense situations. Since such situations are disturbing and add suffering to the illness and hospitalization experience, one of the main purposes of nursing is alleviating the client's emotional suffering - the emotional labour. This algorithm was applied in three paediatric services where its importance emerged. We intend to develop an action research project to validate and apply the algorithm in a specific context.

As a new, singular approach, it will allow the individualization, guidance and adjustment of nursing care in an intentional way, addressed to a specific population; hence we believe that this will be a useful tool for teenagers' nursing care.

\section{OC28 - Effect of mode of delivery on early oral colonization and childhood dental caries: a systematic review}

Celeste Antão (Portugal)2 ${ }^{2}$ Cristina Teixeira (Portugal)1;

Maria José Gomes (Portugal)²

${ }^{1}$ EPI Unit, Public Health Institute, University of Porto, Portugal; ${ }^{2}$ Núcleo de Investigação e Intervenção do Idoso, Polythecnic Institute of Bragança, Bragança, Portugal

Theme: Multidisciplinary team working.

Keywords: Early childhood caries, early oral colonization, acquisition of oral microflora, mode of delivery.

Background: Oral colonization starts at birth by vertical transmission.

Objective: To determine whether mode of delivery influences the oral colonization of infants and contributes to the risk of childhood dental caries.

Methods: A systematic review was conducted in the electronic database Web of Science for articles published from January 1995 to December 2015 by using a set of keywords. 
Results: From 2,644 citations identified through electronic search, ten studies met the inclusion criteria. According to the studies mode of delivery influences oral microbial density, oral microbial profile and the timing of oral colonization by cariogenic microbiota. However, there are no consistent results concerning either the prevalence of children harboring cariogenic microbiota or the prevalence of early childhood caries by mode of delivery.

Conclusion: Mode of delivery influences early oral colonization. However, it seems that other determinants rather than mode of delivery could be major contributors to the development of early childhood caries.

\title{
OC29 - Caregiver burden in childhood asthma
}

\author{
Ayfer Ekim (Turkey) ${ }^{1}$ \\ ${ }^{1}$ Istanbul Bilgi University School of Health Sciences Department of Nursing
}

Theme: Parenting/parenthood.

Keywords: Burden, caregiver, childhood asthma, parent.

Introduction: As delivering care is an inherent part of becoming parents, providing highlevel care to the child with health problems might be a burden for the caregiver.

\begin{abstract}
Aim: This systematic review investigated the effects of caregiver burden of parents who are primarily responsible for the caring of children with asthma.
\end{abstract}

Methods: PubMed, CINAHL, Web of Science and Scopus databases were searched for the studies published between 2000-2015. Key search terms used were 'caregiver', 'burden, 'childhood asthma' and 'parent'.

Results: A total of 23 studies were examined for this review.

Discussion: Providing care to the child with asthma affects parents as caregivers negatively. Caregivers of the children with asthma often experience anxiety, fear, disappointment, grief, physical distress and low quality of life as the outcomes of caregiving burden.

Conclusions: It is absolutely crucial to determine caregiver burden risks and protective factors since wellness level of caregivers affect the asthma management practices and decisions.

\section{OC30 - Fracture reduction with nitrous oxide at the children's emergency department shortens the length of stay and reduces the use of full anaesthesia in the operating department}

Sigrid Lang (Sweden)ㄹ; Anna-Pia Wentzel (Sweden)루

Malin Ekstrom (Sweden) ${ }^{1}$

${ }^{1}$ Queen Silvias Hospital, Gothenburg

Theme: Accreditation and quality improvement. 